\title{
ON THE HIGHER WHITEHEAD GROUPS OF A BIEBERBACH GROUP
}

\author{
BY
}

\author{
ANDREW J. NICAS ${ }^{1}$
}

\begin{abstract}
Let $\Gamma$ be a Bieberbach group, i.e. the fundamental group of a compact flat Riemannian manifold. In this paper we show that if $p>2$ is a prime, then the $p$-torsion subgroup of $\mathrm{Wh}_{i}(\Gamma)$ vanishes for $0 \leq i \leq 2 p-2$, where $\mathrm{Wh}_{i}(\Gamma)$ is the $i$ th higher Whitehead group of $\Gamma$. The proof involves Farrell and Hsiang's structure theorem for Bieberbach groups, parametrized surgery, pseudoisotopy, and Waldhausen's algebraic $K$-theory of spaces.
\end{abstract}

0. Introduction. A closed aspherical manifold is a closed manifold whose universal covering space is contractible. There is the following conjecture concerning the algebraic $K$-theory of such manifolds.

Conjecture. Let $\Gamma$ be the fundamental group of a closed aspherical manifold. Then $\mathrm{Wh}_{i}(\Gamma)=0$ for $i \geq 0$, where $\mathrm{Wh}_{i}$ is the $i$ th higher Whitehead group of $\Gamma$.

In particular, if $\Gamma$ is a Bieberbach group, i.e. the fundamental group of a compact flat Riemannian manifold, Farrell and Hsiang have shown $\mathrm{Wh}_{i}(\Gamma)=0, i=0,1$ [5], and $\mathrm{Wh}_{i}(\Gamma) \otimes Q=0$ for $i \geq 0[\mathbf{6}]$. In $\left[\mathbf{1 2}\right.$ and 13] it was shown that $\mathrm{Wh}_{i}(\Gamma) \otimes \mathbf{Z}\left[\frac{1}{2}\right]=$ $0, i=2,3$.

In this paper the techniques of $[\mathbf{5}, \mathbf{1 2}, \mathbf{1 3}]$ and Waldhausen's algebraic $K$-theory of topological spaces [14] are used to prove

MAIN THEOREM. Let $\Gamma$ be a Bieberbach group and $p>2$ a prime. Then

$$
\mathrm{Wh}_{j}(\Gamma)_{(p)}=0 \quad \text { for } 0 \leq j \leq 2 p-2 \text {. }
$$

The notation $A_{(p)}$ is used here to denote the $p$-torsion subgroup of the abelian group $A$. The main technical ingredient is Proposition 1.5 which asserts a certain vanishing property for the transfer map of $\pi_{j}\left(\mathrm{Wh}^{\mathrm{PL}}(M)\right)$ for compact flat Riemannian $M$. Using a different approach, Frank Quinn has announced that he has developed new techniques which will prove $\mathrm{Wh}_{i}(\Gamma)=0, i \geq 0$, for a Bieberbach group $\Gamma$.

1. A vanishing lemma for the transfer. Recall from [12 and 13$]$ the following

DEFINITION 1.1. Let COV be the category whose objects are closed manifolds and whose morphisms $\tilde{M} \rightarrow M$ are covering projections. Suppose $F: \mathrm{COV} \rightarrow \mathrm{Ab}$ is a contravariant functor where $\mathrm{Ab}$ is the category of abelian groups. $F$ is said to satisfy the vanishing condition for flat manifolds if for each compact flat Riemannian manifold $M$ and $b \in F(M)$ there is a positive integer $N_{b}$ (depending on $b$ ) such

Received by the editors November 28, 1983 and, in revised form, May 18, 1984.

1980 Mathematics Subject Classification. Primary 18F25; Secondary 57N37, 57R65.

${ }^{1}$ Supported by a NSERC University Research Fellowship and grant U0312. 
that if $\phi: M \rightarrow M$ is $s$-expansive (see [5] for the definition) and $s>N_{b}$, then $F(\phi)(b)=0$.

Let $M$ be a smooth manifold with (a possibly empty) boundary $\partial M$. Let $P(M, \partial)$ be the simplicial group of topological pseudoisotopies of $M$ relative to $\partial M$ and $P_{\text {DIFF }}(M, \partial)$ be the simplicial group of smooth pseudoisotopies of $M$ relative to $\partial M . P(M, \partial)$ and $P_{\mathrm{DIFF}}(M, \partial)$ have natural involutions; furthermore, there are "suspension" maps

$$
\Sigma: P(M, \partial) \rightarrow P(M \times I, \partial) \quad \text { and } \quad \Sigma: P_{\mathrm{DIFF}}(M, \partial) \rightarrow P_{\mathrm{DIFF}}(M \times I, \partial)
$$

(see [7]). K. Igusa has proved the stability theorem for smooth pseudoisotopy [8]. This result states that there is a function $\phi_{1}: \mathbf{Z}^{+} \rightarrow \mathbf{Z}^{+}\left(\mathbf{Z}^{+}\right.$is the set of nonnegative integers) satisfying the inequality $\phi_{1}(m) \geq(m-10) / 7$ such that, for any smooth manifold $M^{n}$ with $n \geq 6, \Sigma: P_{\text {DIFF }}(M, \partial) \rightarrow P_{\text {DIFF }}(M \times I, \partial)$ is $k$-connected for $k \leq \phi_{1}(n)$. It follows from the results of $[2]$ that there is a function $\phi_{2}: \mathbf{Z}^{+} \rightarrow \mathbf{Z}^{+}$with the property $\phi_{2}(m) \rightarrow \infty$ as $m \rightarrow \infty$ such that for any smoothable topological manifold $M^{n}$ with $n \geq 6$ the map $\Sigma: P(M, \partial) \rightarrow$ $P(M \times I, \partial)$ is $k$-connected for $k \leq \phi_{2}(n)$.

If $N$ is a manifold and $p: \tilde{N} \rightarrow N$ a covering projection, then there is a transfer $\operatorname{map} \operatorname{tr}(p): P(N, \partial) \rightarrow P\left(\tilde{N}, p^{-1}(\partial N)\right)$ defined using the homotopy lifting property (see [12]); furthermore, $\operatorname{tr}(p)$ commutes (up to homotopy) with the involution. The notation $\pi_{k}^{+}(P(N, \partial))$ and $\pi_{k}^{-}(P(N, \partial))$ will be used to denote respectively the positive and negative eigenspaces of the involution on $\pi_{k}(P(N, \partial))$. The following theorem was proved in $[\mathbf{1 3}]$.

PROPOSITION 1.2. $M \rightarrow \pi_{k}^{-}(P(M)) \otimes \mathbf{Z}\left[\frac{1}{2}\right]$ satisfies the vanishing condition for flat manifolds provided $\operatorname{dim}(M) \geq 6$ and $k<\phi_{2}(\operatorname{dim}(M))$.

There is an inclusion map $i: M \rightarrow M \times S^{1}, i(m)=(m, 1)$, where $S^{1}$ is the unit circle inside the complex numbers. Recall from [7] that $i$ induces a map $i_{*}: P(M) \rightarrow P\left(M \times S^{1}\right)$ defined as follows: Let $J \subset S^{1}$ be a closed arc with $1 \in$ interior $(J)$. Then $i_{*}$ is obtained from the composite

$$
P(M) \stackrel{\Sigma}{\rightarrow} P(M \times J) \stackrel{h}{\rightarrow} P\left(M \times S^{1}\right),
$$

where $h$ is defined by extension. By $[3$, Chapter 6 , Theorem 2], $h$ induces a split injection in homotopy. Hence if $k<\phi_{2}(\operatorname{dim}(M))$, the map $i^{*}: \pi_{k}(P(M)) \rightarrow$ $\pi_{k}\left(P\left(M \times S^{1}\right)\right)$ is a split injection; furthermore, $i^{*}$ anticommutes with the involution since $\Sigma$ does $\left[\mathbf{7}\right.$, Appendix I]. In particular, $i^{*}$ induces an injection $i^{*}: \pi_{k}^{+}(P(M)) \rightarrow$ $\pi_{k}^{-}\left(P\left(M \times S^{1}\right)\right)$.

Now suppose $M^{n}$ is a compact flat Riemannian manifold with $n \geq 6$ and suppose $k<\phi_{2}(n)$. Let $b \in \pi_{k}^{+}(P(M)) \otimes \mathbf{Z}\left[\frac{1}{2}\right]$. Noting that $M \times S^{1}$ is also a compact flat Riemannian manifold, Proposition 1.2 implies that there is a positive integer $N$ such that if $\tilde{\phi}: M \times S^{1} \rightarrow M \times S^{1}$ is $s$-expansive and $s>N$, then $\operatorname{tr}(\tilde{\phi})\left(i_{*}(b)\right)=0$ in $\pi_{k}^{-}\left(P\left(M \times S^{1}\right)\right) \otimes \mathbf{Z}\left[\frac{1}{2}\right]$. Suppose $\phi: M \rightarrow M$ is $s$-expansive and $s>N$. Let $\alpha_{s}: S^{1} \rightarrow S^{1}$ be the map $\alpha_{s}(z)=z^{s}$. Note that $\left(\phi \times \alpha_{s}\right) i=i \phi$. There is a commutative diagram:

$$
\begin{array}{cccc}
\pi_{k}^{+}(P(M)) \otimes \mathbf{Z}\left[\frac{1}{2}\right] & \rightarrow & \pi_{k}^{-}\left(P\left(M \times S^{1}\right)\right) \otimes \mathbf{Z}\left[\frac{1}{2}\right] \\
\operatorname{tr}(\phi) \uparrow & & \uparrow \operatorname{tr}\left(\phi \times \alpha_{s}\right) \\
\pi_{k}^{+}(P(M)) \otimes \mathbf{Z}\left[\frac{1}{2}\right] & \rightarrow & \pi_{k}^{-}\left(P\left(M \times S^{1}\right)\right) \otimes \mathbf{Z}\left[\frac{1}{2}\right]
\end{array}
$$


Since $\phi \times \alpha_{s}$ is $s$-expansive and $\operatorname{tr}\left(\phi \times \alpha_{s}\right)\left(i_{*}(b)\right)=0$, the injectivity of $i_{*}$ implies $\operatorname{tr}(\phi)(b)=0$. This proves

PROPOSITION 1.3. $M \rightarrow \pi_{k}^{+}(P(M)) \otimes \mathbf{Z}\left[\frac{1}{2}\right]$ satisfies the vanishing condition or flat manifolds provided $\operatorname{dim}(M) \geq 6$ and $k<\phi_{2}(\operatorname{dim}(M))$.

Since

$$
\pi_{k}(P(M)) \otimes \mathbf{Z}\left[\frac{1}{2}\right]=\pi_{k}^{+}(P(M)) \otimes \mathbf{Z}\left[\frac{1}{2}\right] \oplus \pi_{k}^{-}(P(M)) \otimes \mathbf{Z}\left[\frac{1}{2}\right],
$$

Propositions 1.2 and 1.3 imply

Proposition 1.4. $M \rightarrow \pi_{k}(P(M)) \otimes \mathbf{Z}\left[\frac{1}{2}\right]$ satisfies the vanishing condition for flat manifolds provided $\operatorname{dim}(M) \geq 6$ and $k<\phi_{2}(\operatorname{dim}(M))$.

The space $P^{s}(M)$ of stable pseudoisotopies of $M$ is defined to be the direct limit of the sequence $P(M) \stackrel{\Sigma}{\rightarrow} P(M \times I, \partial) \stackrel{\Sigma}{\rightarrow} \cdots$. By the stability theorem for pseudoisotopies the natural map $P(M) \rightarrow P^{s}(M)$ is $k$-connected for $k \leq \phi_{2}(\operatorname{dim} M)$.

Waldhausen $[14,16]$ has defined a functor $X \rightarrow \mathrm{Wh}^{\mathrm{PL}}(X)$ from the category of simplicial sets to the category of infinite loop spaces with the property that if $X$ is a piecewise linear manifold, then $\mathrm{Wh}^{\mathrm{PL}}(X)$ is homotopy equivalent to a canonical 2-fold deloop of the space $P_{\mathrm{PL}}^{S}(X)$ of stable piecewise linear pseudoisotopies of $X$. Note that by $[\mathbf{1}] P_{\mathrm{PL}}^{S}(X)$ is homotopy equivalent to $P^{s}(X)$ for triangulable $X$ with $\operatorname{dim}(X) \geq 6$.

Proposition 1.5. For all $k \geq 0, M \rightarrow \pi_{k}\left(\mathrm{Wh}^{\mathrm{PL}}(M)\right) \otimes \mathbf{Z}\left[\frac{1}{2}\right]$ satisfies the vanishing condition for flat manifolds.

PROOF. By the preceding discussion and Proposition 1.4 we have that $M \rightarrow$ $\pi_{k}\left(\mathrm{Wh}^{\mathrm{PL}}(M)\right) \otimes \mathbf{Z}\left[\frac{1}{2}\right]$ satisfies the vanishing condition for flat manifolds provided $\operatorname{dim}(M) \geq 6$ and $k<\phi_{2}(\operatorname{dim}(M))$. Let $M^{n}$ be any compact flat Riemannian manifold and let $r$ be sufficiently large so that $n+r \geq 6$ and $k<\phi_{2}(r+n)$. Let $\alpha_{s}: S^{1} \rightarrow S^{1}$ be the map $\alpha_{s}(z)=z^{s}$ and $i: M \rightarrow M \times\left(S^{1}\right)^{r}$ the inclusion $i(m)=(m, 1, \ldots, 1)$. Suppose $\phi: M \rightarrow M$ is $s$-expansive. Then $\phi \times\left(\alpha_{s}\right)^{r}$ is $s$-expansive and there is a commutative diagram:

$$
\begin{array}{ccc}
\pi_{k}\left(\mathrm{Wh}^{\mathrm{PL}}(M)\right) & \stackrel{i_{*}}{\rightarrow} & \pi_{k}\left(\mathrm{Wh}^{\mathrm{PL}}\left(M \times\left(S^{1}\right)^{r}\right)\right) \\
\operatorname{tr}(\phi) \uparrow & & \uparrow \operatorname{tr}\left(\phi \times \alpha_{s}^{r}\right) \\
\pi_{k}\left(\mathrm{Wh}^{\mathrm{PL}}(M)\right) & \stackrel{i_{*}}{\rightarrow} & \pi_{k}\left(\mathrm{Wh}^{\mathrm{PL}}\left(M \times\left(S^{1}\right)^{r}\right)\right)
\end{array}
$$

Since $i_{*}$ is a (split) injection the proposition follows.

2. Proof of the Main Theorem. Waldhausen has defined a homotopy functor $X \rightarrow A(X)$ from the category of simplicial sets to infinite loop spaces $[14,16]$. There is a homotopy fibration

$$
h(X ; A(*)) \rightarrow A(X) \rightarrow \mathrm{Wh}^{\mathrm{PL}}(X) .
$$

The space $h(X ; A(*))$ is a homology theory with coefficients in $A(*)$ in the sense that $\pi_{i} h(X ; A(*))$ is $h_{i}(X ; A(*))$.

According to $[\mathbf{1 4}, \mathbf{1 5}]$ there is a functor $\pi \rightarrow \mathrm{Wh}(\pi)$ from the category of groups to the category of infinite loop spaces and a homotopy fibration

$$
h(B \pi ; K(Z)) \rightarrow B G L(Z \pi)^{+} \rightarrow \mathrm{Wh}(\pi)
$$


such that:

(i) $h(B \pi ; K(Z))$ is the homology theory with coefficients in the $\Omega$-spectrum $K(Z)$ with zeroth space $B G L(Z)^{+}$and the induced map

$$
h_{i}(B \pi ; K(Z))=\pi_{i} h(B \pi ; K(Z)) \rightarrow \pi_{i} B G L(Z \pi)^{+}=K_{i}(Z \pi)
$$

is Loday's homomorphism $[\mathbf{1 0}]$ and $\pi_{j} \mathrm{Wh}(\pi)=\mathrm{Wh}_{j}(\pi)$.

(ii) There is a natural map of fibrations:

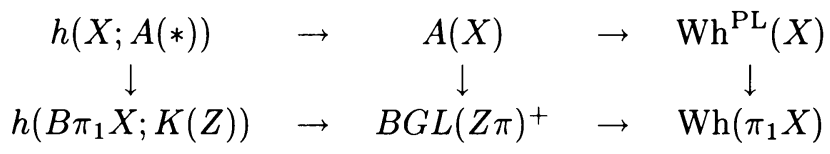

Let $C_{p}$ be the Serre class of abelian groups consisting of those torsion abelian groups having no element with order a positive power of $p$. A map of spaces $f: X \rightarrow Y$ will be called $k$-connected $\bmod C_{p}$ if $f_{*}: \pi_{0} X \rightarrow \pi_{0} Y$ is an isomorphism and, for any basepoint, $f_{*}: \pi_{j} X \rightarrow \pi_{j} Y$ is an isomorphism $\bmod C_{p}$ for $j<k$ and $f_{*}: \pi_{k} X \rightarrow \pi_{k} Y$ is an epimorphism and $\bmod C_{p}$.

LEMMA 2.4. Suppose $p$ is a prime and $X$ is an aspherical space; then the map

$$
\pi_{j} \mathrm{Wh}^{\mathrm{PL}}(X) \rightarrow \mathrm{Wh}_{j}\left(\pi_{1} X\right)
$$

is an isomorphism $\bmod C_{p}$ if $0<j<2 p-2$ and an epimorphism $\bmod C_{p}$ if $j=2 p-2$.

ProOF. Let $Q$ be the functor $\lim _{\longrightarrow} \Omega^{n} \Sigma^{n}$. If $X$ is a space let $\Omega^{*} X$ be the topological monoid of Moore loops on $X$. There is a natural map of $A_{\infty}$ ring spaces (see [9] for the definition):

$$
\theta: Q\left(\Omega^{*} X \cup *\right) \rightarrow Z\left(\Omega^{*} X\right)
$$

where $\Omega^{*} X \cup *$ is the union with the disjoint base point $*$ and $Z\left(\Omega^{*} X\right)$ is the integral monoid ring of $\Omega^{*} X$. According to [14] $\theta$ is 1-connected. Now assume $X$ is aspherical. Then $\Omega^{*} X$ has the homotopy type of a discrete set so that $\pi_{j}\left(Z \Omega^{*} X\right)=$ 0 for $j>0$ and any basepoint. Note that $\pi_{j}\left(Q\left(\Omega^{*} X \cup *\right)\right)$ belongs to $C_{p}$ for $0<j<2 p-3$ since the first $p$-torsion occurs in the stable homotopy of a point $\pi_{j}^{s}$ when $j=2 p-3$. Hence $\theta$ is $(2 p-3)$-connected $\bmod C_{p}$. By the $\bmod C_{p}$ analog of [14, Proposition 1.1] for $A_{\infty}$ ring spaces it follows that

$$
B \widehat{G L}\left(Q\left(\Omega^{*} X \cup *\right)\right)^{+} \rightarrow \widehat{B G L}\left(Z \Omega^{*} X\right)^{+}
$$

is $(2 p-2)$-connected $\bmod C_{p} .\left(B \widehat{G L}\left(Z \Omega^{*} X\right)^{+}\right.$is the $K$-theory of the topological ring $Z \Omega^{*} X$.) Since $\Omega^{*} X$ has the homotopy type of a discrete set we have for $j \geq 1$

$$
\pi_{j} B \widehat{G L}\left(Z \Omega^{*} X\right)^{+}=K_{j}\left(Z \pi_{0} \Omega^{*} X\right)=K_{j}\left(Z \pi_{1} X\right) .
$$

Note that $A(X)$ is (by one of its many definitions) $B \widehat{G L}\left(Q\left(\Omega^{*} X \cup *\right)\right)^{+}$(see [14]). In particular, $A(*) \rightarrow K(Z) \simeq B G L(Z)^{+}$is $(2 p-2)$-connected $\bmod C_{p}$. It follows that if $Y$ is a finite complex, $h(Y ; A(*)) \rightarrow h(Y ; K(Z))$ is $(2 p-2)$-connected $\bmod C_{p}$. A standard diagram chase with the ladder of exact sequence obtained from the map of homotopy fibrations (2.3) yields the lemma.

Let $Z_{\langle p\rangle}$ be the subring of the rationals generated by fractions of the form $a / b$, where $b \neq 0$ and $p$ does not divide $b$. 
PROPOSITION 2.5. Let $\Gamma$ be a Bieberbach group and $p>2$ a prime. Suppose $b \in \mathrm{Wh}_{k}(\Gamma) \otimes \mathbf{Z}_{\langle p\rangle}$, where $0 \leq k \leq 2 p-2$. Then there is an integer $N_{b}$ such that if $\theta: \Gamma \rightarrow \Gamma$ is s-expansive and $s>N_{b}$, then $\operatorname{tr}(\theta)(b)=0$.

Proof. In the cases $k=0,1$ this was proved in [5]. Suppose $\theta: \Gamma \rightarrow \Gamma$ is $s$-expansive (see [5] for the definition). By [4] there is a compact flat Riemannian manifold $M$ with $\pi_{1} M=\Gamma$ and an $s$-expansive map $H: M \rightarrow M$ such that $\theta=$ $H_{*}: \pi_{1} M \rightarrow \pi_{1} M$. The result now follows from Proposition 1.5, Lemma 2.4 and the fact that $\mathrm{Wh}^{\mathrm{PL}}(M) \rightarrow \mathrm{Wh}\left(\pi_{1} M\right)$ is natural with respect to the transfer.

The following weak form of Frobenius induction is valid for $\mathrm{Wh}_{j}(\Gamma)$.

LEMMA 2.6. Let $\Gamma$ be a group, $p: \Gamma \rightarrow G$ an epimorphism to a finite group, $R$ a subring of the rationals and $j \geq 0$. Suppose $b \in \mathrm{Wh}_{j}(\Gamma) \otimes R$ and, for every hyperelementary subgroup $E$ of $G, \operatorname{tr}(b)=0$ where $\operatorname{tr}: \mathrm{Wh}_{j}(\Gamma) \otimes R \rightarrow \mathrm{Wh}_{j}\left(p^{-1}(E)\right) \otimes R$ is the transfer. Then $b=0$.

Proof. For $H \subset G$ there is a long exact sequence from the fibration (2.2):

$$
\begin{aligned}
\rightarrow h_{j}\left(B p^{-1}(H) ; K(Z)\right) & \stackrel{l_{j}}{\rightarrow} K_{j}\left(Z p^{-1}(H)\right) \\
& \left.\stackrel{q_{j}}{\rightarrow} \mathrm{Wh}_{j}\left(p^{-1}(H)\right) \rightarrow \cdots \rightarrow \mathrm{Wh}_{0}\left(p^{-1} H\right)\right) \rightarrow 0 .
\end{aligned}
$$

This sequence remains exact when tensored with $R$. Let

$$
W_{j}(H)=\operatorname{Image}\left(q_{j}\right) \otimes R, \quad V_{j}(H)=\operatorname{ker}\left(l_{j-1}\right) \otimes R \quad\left(\text { set } V_{0}(H)=0\right) .
$$

Then for any $H \subset G$ there is a short exact sequence

$$
0 \rightarrow W_{j}(H) \rightarrow \mathrm{Wh}_{j}\left(p^{-1}(H)\right) \otimes R \rightarrow V_{j}(H) \rightarrow 0 .
$$

According to $[\mathbf{6}], K_{j}\left(Z p^{-1}(H)\right)$ is a Frobenius module over Swan's Frobenius functor $G_{0}(H)$. This action induces an action of $G_{0}(H)$ on $W_{j}(H)$ and consequently $W_{j}$ satisfies hyperelementary induction, in particular, if $C$ is the collection of hyperelementary subgroups of $G$ the product of the transfer ("restriction") maps is injective:

$$
0 \rightarrow W_{j}(G) \rightarrow \prod_{H \in C} W_{j}(H)
$$

Also the product of the transfer ("restriction") maps for $V_{j}$ is injective (compare [11, Proposition 6.2.9]):

$$
0 \rightarrow V_{j}(G) \rightarrow \prod_{H \in C} V_{j}(H)
$$

It follows from (2.7) that the product over $C$ of the transfer maps

$$
\mathrm{Wh}_{j}(\Gamma) \otimes R \rightarrow \prod_{H \in C} \mathrm{Wh}_{j}\left(p^{-1}(H)\right) \otimes R
$$

is injective, which yields the lemma.

The Main Theorem is now easily proved by following the argument of [5] which was successfully copied in [12 and 13] with Proposition 2.5 providing the crucial vanishing result for the transfer and Lemma 2.6 showing Frobenius induction remains valid. We summarize this argument as follows:

If $\Gamma$ is a Bieberbach group and $A \subset \Gamma$ its unique maximal normal abelian subgroup of finite index, then $\Gamma / A$ is called the holonomy group of $\Gamma$ and the rank 
of $\Gamma$ is defined to be the rank of $A$. A homomorphism $f: \Gamma \rightarrow \Gamma$ is said to be $s$-expansive, where $s$ is an integer, if its restriction to $A$ is multiplication by $s$ and it induces the identity map on $\Gamma / A$. If $s$ is a positive integer define $\Gamma_{s}=\Gamma / s A$. Hsiang and Farrell proved the following structure theorem.

THEOREM $2.8[\mathbf{5}]$. Let $\Gamma$ be a Bieberbach group of rank $n$ and holonomy group $G$. Then either:

(1) $\Gamma=\Pi \times_{\alpha} T$; i.e. $\Gamma$ is the semidirect product of subgroups $\Pi$ and $T$, where $\Pi$ is a Bieberbach group of rank $n-1$; or

(2) $\Gamma=B *_{D} C$; i.e. is the amalgamated free product of subgroups of rank $n-1$, where $D$ has index 2 in both $B$ and $C$; or

(3) there is an infinite sequence of positive integers $S_{n}$ with $S_{n} \equiv 1 \bmod |G|(|G|$ $=$ order of $G$ ) such that any hyperelementary subgroup of $\Gamma_{s}$ which projects onto $G$ via the canonical map $\Gamma \rightarrow G$ projects isomorphically to $G$.

The proof that $\mathrm{Wh}_{j}(\Gamma)_{(p)}=0$ for $0 \leq j \leq 2 p-2$ proceeds by induction on $j$, on the rank of $\Gamma$ and on the order of the holonomy group of $\Gamma$. The induction starts with the result $\mathrm{Wh}_{0}(\Gamma)=0$ from [5]. Assuming the induction hypothesis, if we are in the first or second case of the above theorem then the result follows from Waldhausen's theory concerning the Whitehead groups of generalized free products [9] (the Nil terms vanish since the ring $Z \Gamma$ is left-regular and noetherian). In the third case let $b \in \mathrm{Wh}_{j}(\Gamma)_{(p)}$ and $s$ be one of the integer $S_{n}$ given by Theorem 2.8(3) subject to the constraint $s>N_{b}$, where $N_{b}$ comes from Proposition 2.5. Let $p: \Gamma \rightarrow \Gamma_{s}$ be the canonical map. By Lemma 2.6 we will have $b=0$ if $b$ vanishes under the transfer maps associated to the hyperelementary subgroups of $\Gamma_{s}$. If $E$ is a hyperelementary subgroup of $\Gamma_{s}$ which projects via $p$ to a proper subgroup of $G$, then the holonomy group of $p^{-1}(E)$ has order less than the order of $G$; thus, $\mathrm{Wh}_{j}\left(p^{-1}(E)\right)_{(p)}=0$ by the induction hypothesis. Otherwise $E$ projects isomorphically to $G$ by Theorem $2.8(3)$. Since all such subgroups are conjugate [5] it suffices to consider one of them. Let $E=p f(\Gamma)$, where $f: \Gamma \rightarrow \Gamma$ is $s$-expansive. However, $f^{*}(b)=0$ by Proposition 2.5 ; hence, $b$ vanishes under all appropriate transfer maps, completing the proof of the Main Theorem.

REMARKS. (1) As a corollary of the proof of Proposition 2.5 and the Main Theorem we have

$$
\begin{aligned}
\pi_{j} P^{s}(M)_{(p)} & =\pi_{j+2} \mathrm{Wh}^{\mathrm{PL}}(M)_{(p)} \\
& =\mathrm{Wh}_{j+2}\left(\pi_{1} M\right)_{(p)} \text { for } 0 \leq j \leq 2 p-5 \text { and } p>2 \\
& =0
\end{aligned}
$$

where $M$ is a compact flat Riemannian manifold. As a consequence, for $p>2$, the $p$-torsion of $\pi_{j} \operatorname{TOP}(M)$ vanishes in the range $0 \leq j \leq 2 p-5$ and $j<\phi_{2}(\operatorname{dim}(M))$, where $\operatorname{TOP}(M)$ is the group of homeomorphisms of $M$ (compare $[\mathbf{1 3}, \S 2]$ ).

(2) If $\pi_{k}\left(\mathrm{Wh}^{\mathrm{PL}}(M)\right) \otimes \mathbf{Z}\left[\frac{1}{2}\right] \rightarrow \mathrm{Wh}_{k}\left(\pi_{1} M\right) \otimes \mathbf{Z}\left[\frac{1}{2}\right]$ is onto for $k \geq 0$ and for compact flat Riemannian $M$, then the techniques used in this paper show $\mathrm{Wh}_{k}(\Gamma) \otimes$ $\mathbf{Z}\left[\frac{1}{2}\right]=0, k \geq 0$, for any Bieberbach group. 


\section{REFERENCES}

1. D. Burghelea and R. Lashof, The homotopy type of the space of diffeomorphisms. II, Trans. Amer. Math. Soc. 196 (1974), 37-50.

2. D. Burghelea and R. Lashof, Stability of concordances and the suspension homomorphism, Ann. of Math. (2) 105 (1977), 449-472.

3. D. Burghelea, R. Lashof and M. Rothenberg, Groups of automorphisms of manifolds, Lecture Notes in Math., Vol. 473, Springer-Verlag, New York, 1975.

4. D. Epstein and M. Shub, Expanding endomorphisms of flat manifolds, Topology 7 (1968), 139141.

5. F. Farrell and W.-C. Hsiang, The topological Euclidean space form problem, Invent. Math. 45 (1978), 181-192.

6. _ - On the rational homotopy groups of the diffeomorphism groups of discs, spheres, and aspherical manifolds, Proc. Sympos. Pure Math., vol. 32, Amer. Math. Soc., Providence, R.I., 1978, pp. 325-338.

7. A. Hatcher, Concordance spaces, higher simple homotopy theory and applications, Proc. Sympos. Pure Math., vol. 32, Amer. Math. Soc., Providence, R.I., 1978, pp. 3-22.

8. K. Igusa, Stability of pseudoisotopies, Aarhus lecture notes, 1981.

9. __ On the algebraic $K$-theory of $A_{\infty}$-ring spaces, Lecture Notes in Math., vol. 967, SpringerVerlag, New York, 1982, pp. 146-194.

10. J. Loday, K-theorie algébrique et représentations de groups, Ann. Sci. École Norm. Sup (4) 9 (1976), 306-377.

11. A. Nicas, Induction theorems for groups of homotopy manifold structures, Mem. Amer. Math. Soc. No. 267 (1982).

12. __ On $\mathrm{Wh}_{2}$ of a Bieberbach group, Topology (to appear).

13. _ , On $\mathrm{Wh}_{3}$ of a Bieberbach group, Math. Proc. Cambridge Philos. Soc. 95 (1984), 55-60.

14. F. Waldhausen, Algebraic K-theory of topological spaces. I, Proc. Sympos. Pure Math., vol. 32, Amer. Math. Soc., Providence, R.I., 1978, pp. 35-60.

15. __ Algebraic K-theory of generalized free products, Ann. of Math. (2) 108 (1978), 135-256.

16. __ Algebraic K-theory of spaces-a manifold approach, Current Trends in Algebraic Topology, CMS Conf. Proc., vol. 2, Part I, Amer. Math. Soc., Providence, R.I., 1982, pp. 141-184.

Department of MATHEMATiCs, University of TORONTO, TORONTO, Ontario M5S 1A1, CANADA 